\title{
Notch Signaling Controls Oligodendrocyte Regeneration in the Injured Telencephalon of Adult Zebrafish
}

\author{
Hwan-Ki Kim ${ }^{1}$, Dong-won Lee ${ }^{1}$, Eunmi Kim ${ }^{1}$, Inyoung Jeong ${ }^{1}$, Suhyun Kim ${ }^{1 *}$, \\ Bum-Joon $\mathrm{Kim}^{2 *}$ and Hae-Chul Park ${ }^{1 *}$ \\ ${ }^{1}$ Department of Biomedical Sciences, College of Medicine, Korea University, \\ ${ }^{2}$ Department of Neurosurgery, Korea University Ansan Hospital, Ansan 15355, Korea
}

The myelination of axons in the vertebrate nervous system through oligodendrocytes promotes efficient axonal conduction, which is required for the normal function of neurons. The central nervous system (CNS) can regenerate damaged myelin sheaths through the process of remyelination, but the failure of remyelination causes neurological disorders such as multiple sclerosis. In mammals, parenchymal oligodendrocyte progenitor cells (OPCs) are known to be the principal cell type responsible for remyelination in demyelinating diseases and traumatic injuries to the adult CNS. However, growing evidence suggests that neural stem cells (NSCs) are implicated in remyelination in animal models of demyelination. We have previously shown that olig2 $2^{+}$radial glia (RG) have the potential to function as NSCs to produce oligodendrocytes in adult zebrafish. In this study, we developed a zebrafish model of adult telencephalic injury to investigate cellular and molecular mechanisms underlying the regeneration of oligodendrocytes. Using this model, we showed that telencephalic injury induced the proliferation of olig2 $2^{+}$RG and parenchymal OPCs shortly after injury, which was followed by the regeneration of new oligodendrocytes in the adult zebrafish. We also showed that blocking Notch signaling promoted the proliferation of olig2 ${ }^{+}$RG and OPCs in the normal and injured telencephalon of adult zebrafish. Taken together, our data suggest that Notch-regulated proliferation of olig2 ${ }^{+}$RG and parenchymal OPCs is responsible for the regeneration of oligodendrocytes in the injured telencephalon of adult zebrafish.

Key words: Neural stem cells, Oligodendroglia, Regeneration, Telencephalon, Wounds and injuries, Zebrafish

\section{INTRODUCTION}

Oligodendrocytes are glial cells that myelinate axons in the central nervous system (CNS) to provide electrical insulation for axonal conduction. Oligodendrocytes also play a crucial role in promoting neuronal survival by providing metabolic support, in-

\footnotetext{
Submitted October 13, 2020, Revised November 23, 2020,

Accepted November 24, 2020

* To whom correspondence should be addressed.

Hae-Chul Park, TEL: 82-31-412-6713, FAX: 82-31-412-6729

e-mail:hcpark67@korea.ac.kr

Bum-Joon Kim, TEL: 82-31-412-5050, FAX: 82-31-412-5054

e-mail:nsbjkim@korea.ac.kr

Suhyun Kim, TEL: 82-31-412-6725, FAX: 82-31-412-6729

e-mail:dieslunae@naver.com
}

cluding the supply of lactate to neurons [1]. Various factors including genetics, immune system dysfunction, and traumatic injury can induce demyelination, which can be restored through oligodendrocytes. However, persistent demyelination and the failure of remyelination may cause several neurological disorders, such as multiple sclerosis [2].

Severe traumatic injury to the adult mammalian CNS has catastrophic effects, after which significant regeneration does not occur. In contrast, adult zebrafish possess a remarkable ability to regenerate neurons in the injured CNS. Previous studies have shown that radial glia (RG) detected using markers such as BLBP, GFAP, and S100B exist in the ventricular zone (VZ) and function as neural precursors to generate neurons in the telencephalon of adult zebrafish [3-7]. Injury of the telencephalon induces the proliferation of RGs in the VZ of the injured hemisphere following
Copyright $\odot$ Experimental Neurobiology 2020. www.enjournal.org
This is an Open Access article distributed under the terms of the Creative Commons Attribution Non-Commercial License (http://creativecommons.org/licenses/by-nc/4.0) which permits unrestricted non-commercial use, distribution, and reproduction in any medium, provided the original work is properly cited. 
active neurogenesis for neuronal regeneration [8-11]. In addition, lineage-tracing studies of RG using retroviral and lentiviral vectors and the Cre-loxP system in the normal and injured telencephalon, respectively, have provided direct evidence that RG can act as neural stem cells to generate new neurons in adult zebrafish $[8,12]$. However, the cellular and molecular mechanisms underlying the regeneration of oligodendrocytes in the injured brain are largely unknown.

We previously reported that regulatory DNA from the olig2 locus in transgenic zebrafish embryos drives the expression of enhanced green fluorescent protein (EGFP) in RGs of the pMN precursor domain of the ventral spinal cord and their descendent motor neurons and oligodendrocyte progenitor cells (OPCs), which migrate and divide to produce cells of the oligodendrocyte lineage only [13-15]. Additionally, we showed that oligodendrocytes are continuously generated from the post-embryonic phase to the adult spinal cord and that olig $2^{+}$RG have the potential to function as neural stem cells to produce oligodendrocytes in the post-embryonic spinal cord [16]. The current study aimed to develop a zebrafish model of adult telencephalic injury to investigate the cellular and molecular mechanisms underlying the regeneration of oligodendrocytes. Using this model, we aimed to reveal regenerative processes of oligodendrocytes from olig $2^{+} \mathrm{RG}$ and underlying mechanisms in the injured telencephalon. The study findings would provide a novel zebrafish disease model for studying oligodendrocyte regeneration.

\section{MATERIALS AND METHODS}

\section{Zebrafish line and ethics}

The following transgenic lines were used: $\operatorname{Tg}($ olig2:EGFP) [13] and $\operatorname{Tg}(\mathrm{mbp}: E G F P)$ [17]. Adult animals were housed in a 14-h light and 10-h dark cycle. All experimental procedures were approved by the Korea University Institutional Animal Care \& Use Committee and performed in accordance with the animal experiment guidelines of the Korea National Veterinary Research and Quarantine Service.

\section{Drug treatment}

For LY-411575 treatment, the following stock solution was made and stored at $-20^{\circ} \mathrm{C}: 10 \mathrm{mM} \mathrm{LY}-411575$ (medchemexpress, HY50752) in dimethyl sulfoxide (DMSO); this was added to the swimming water at a temperature of $28^{\circ} \mathrm{C}$ and at a final concentration of $10 \mu \mathrm{M}$ for 4 days. The control fish were placed in water with $0.1 \%$ DMSO.

\section{Stab wound injury}

Adult male fish were 6 10 months old. Fish were anesthetized using Tricaine (Sigma). An insulin syringe (31 gauge, outer diameter: $250 \mu \mathrm{m}$ ) was pushed $\sim 5 \sim 7 \mathrm{~mm}$ deep along the rostrocaudal body axis until it reached the caudal part of the telencephalon [8].

\section{Bromodeoxyuridine (BrdU) labeling}

To label newborn cells in the adult zebrafish telencephalon, adult zebrafish were placed in water containing 10 mM 5-bromo-2'deoxyuridine (BrdU, Roche Holding AG, Basel, Switzerland, 10280879001 ) for 4 days prior to being released into fresh water.

\section{Immunohistochemistry}

Adult zebrafish brains were fixed in 4\% paraformaldehyde prior to being embedded in 1.5\% agar blocks containing 5\% sucrose and equilibrated in $30 \%$ sucrose solution. The frozen blocks were sliced into $10-\mu \mathrm{m}$ sections using a cryostat microtome, and the transverse sectioned slices were mounted on glass slides. Subsequently, the zebrafish were processed for immunohistochemistry. The following primary antibodies were used: rabbit anti-BrdU (1:200, Rockland, cat \# 600-401-C29, RRID: AB_10893609), chicken antiGFP (1:200, Abcam, cat \# ab6556, RRID: AB_300798), mouse anti-proliferating cell nuclear antigen (PCNA) (1:200, DAKO, cat \# M0879, RRID: AB_2313584), rabbit anti-BLBP (1:1,000, Abcam, cat \# ab27171, RRID: AB_869739), rabbit anti-S100ß (1:200, DAKO, cat \# Z031101, RRID: AB_10013383), and mouse antiGFAP (1:250, ZIRC, cat \# Zrf-1, RRID: AB_10013806). For fluorescent detection of antibody labeling, we used Alexa Fluor 488-, 568-, and 647-conjugated secondary antibodies (1:1,000, Molecular Probes).

\section{Quantifications and statistical analyses}

All results are expressed as mean \pm standard error of the mean (SEM). Ten micrometer-thick sections of the adult telencephalon were used for the statistical analyses (approximately 10 sections per slide). p-values were determined with GraphPad Prism (GraphPad Software, San Diego, CA, USA) using the Mann-Whitney $U$ test and one-way analysis of variance followed by the Kruskal-Wallis test. Error bars represent SEM. Significance is denoted with asterisks as follows: ${ }^{* * *} \mathrm{p}<0.0001 ;{ }^{* * *} \mathrm{p}<0.001 ;{ }^{* *} \mathrm{p}<0.01$; and ${ }^{*} \mathrm{p}<0.05$.

\section{RESULTS AND DISCUSSION}

\section{olig ${ }^{+} R G$ exist in the telencephalic ventricular zone of adult zebrafish}

Previously, we have shown that olig2 ${ }^{+}$RG have the hallmarks of stem cells in the post-embryonic spinal cord of zebrafish [16]. 


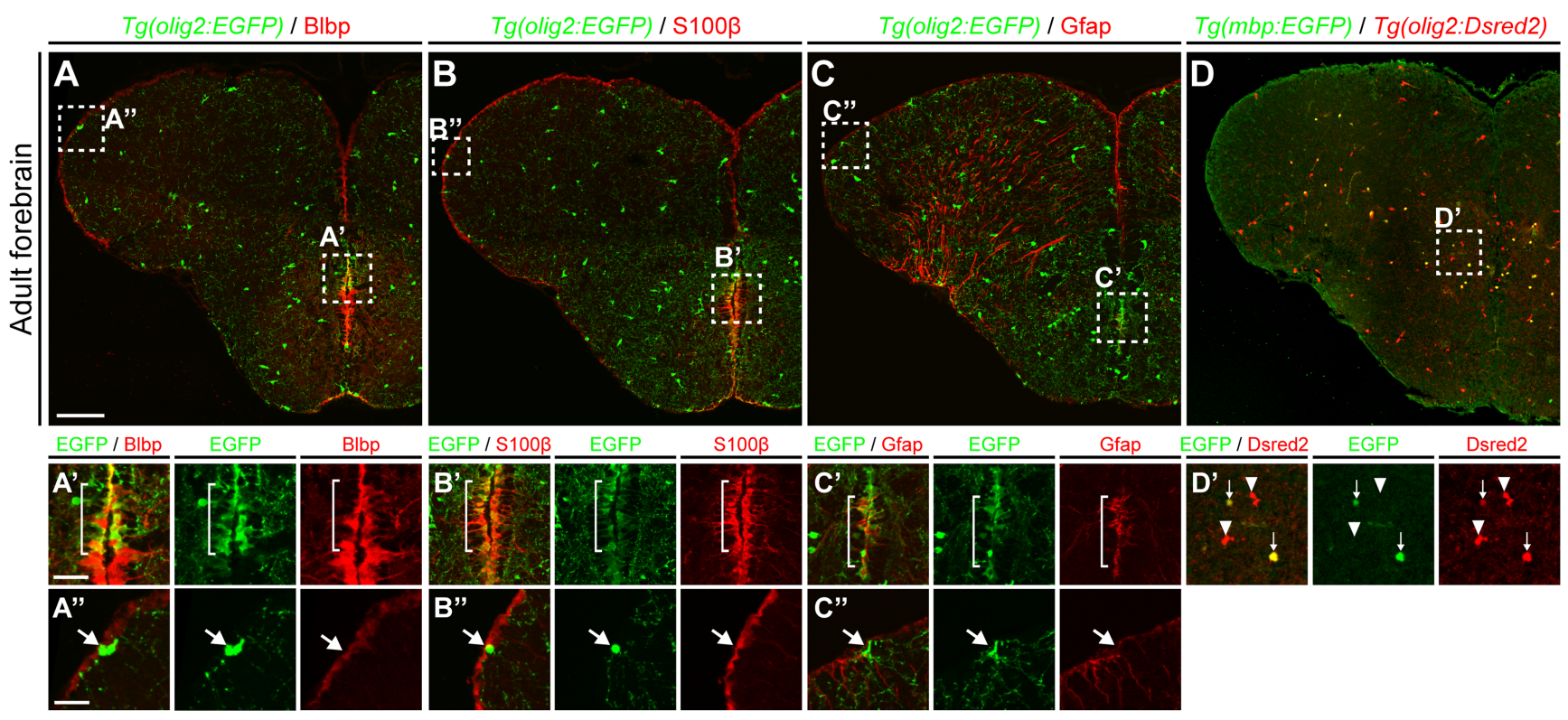

Fig. 1. olig2 ${ }^{+}$RG in the telencephalic ventricular zone of adult zebrafish. All panels show transverse sections of the telencephalon of adult zebrafish, with the dorsal side at the top. (A C) Labeling of $\operatorname{Tg}($ olig2:EGFP) zebrafish with various RG markers: anti-BLBP, -S100 $\beta$, and -GFAP antibodies. Boxed areas indicate the MVZ ( $\left.A^{\prime} \sim C^{\prime}\right)$ and LVZ (A" $\left.\sim C^{\prime \prime}\right)$ of the telencephalon. The bracketed area in $\left(A^{\prime} \sim C^{\prime}\right)$ indicates the MVZ in which olig2 $2^{+} \mathrm{RG}$ exist. (A" $\left.\sim C^{\prime \prime}\right)$ arrows indicate olig $2^{+}$non-RGs in the LVZ. Seven zebrafish brains were analyzed in each indicated group and representative images were presented. (D D') Identification of mature oligodendrocytes in the telencephalic parenchymal region of the adult $\operatorname{Tg}($ olig2:Dsred2)/Tg(mbp:EGFP) zebrafish. The boxed area in (D') indicates the parenchymal region showing oligodendrocyte lineage cells. Arrows indicate $m b p$ :EGFP $/ o$ lig2:Dsred ${ }^{+}$mature oligodendrocytes, and arrowheads indicate mbp:EGFP/olig2:Dsred ${ }^{+}$OPCs ( $\mathrm{n}=8$ sections from one zebrafish). Scale bars: A D, $100 \mu \mathrm{m}$; A $\sim \mathrm{C}^{\prime}, 25 \mu \mathrm{m}$. GFAP, glial fibrillary acidic protein; EGFP, enhanced green fluorescent protein; LVZ, lateral ventricular zone; MVZ, medial ventricular zone; OPCs, oligodendrocyte progenitor cells; RG, radial glia.

We, therefore, hypothesized that olig $2^{+} \mathrm{RG}$ are responsible for the regeneration of oligodendrocytes in the adult CNS and initially investigated the existence of olig $2^{+} \mathrm{RG}$ in the telencephalon of adult $\operatorname{Tg}($ olig2:EGFP $)$ zebrafish, which express EGFP under the control of the olig2 promoter [13]. Immunohistochemical analysis of the adult zebrafish telencephalon with BLBP, S100 $\beta$, and GFAP antibodies that mark RG $[4,6,18-20]$, revealed that olig2 ${ }^{+}$RG exist in the medial ventricular zone (MVZ) of the adult telencephalon (Fig. $\left.1 \mathrm{~A} \sim \mathrm{C}^{\prime}\right)$. However, we did not detect any olig2 expression in RGs of the lateral ventricular zone ( $\mathrm{LVZ}$; $\mathrm{A} \sim \mathrm{C}, \mathrm{A} \sim \mathrm{C}$ "). Consistent with a previous report that shows that olig $2^{+}$cells are oligodendrocyte lineage cells including OPCs and mature oligodendrocytes in the telencephalon [21], olig2:EGFP expression was detected in parenchymal mature oligodendrocytes, which express mbp:EGFP [17] in the telencephalon of the adult $\operatorname{Tg}(m b p$ :EGFP); $\operatorname{Tg}$ (olig2:Dsred2) zebrafish (Fig. 1D, D').

\section{Olig2 ${ }^{+}$RGs and OPCs are responsible for the regeneration of oligodendrocytes in the injured telencephalon}

To test the response of olig2 $2^{+} \mathrm{RG}$ and parenchymal OPCs to invasive injury in the telencephalon of adult zebrafish, we inserted a 31-gauge needle from the anterior side of the telencephalon to generate reproducible stab wound injuries (Fig. 2A). The size of the resultant lesion was consistent across the brains that were dissected after the injury ( $n=15$; Fig. $2 \mathrm{C}$ ). To examine cell proliferation induced by injury to the telencephalon, we analyzed dividing cells 4 days post-lesion ( $\mathrm{dpl}$ ) based on their PCNA expression. In the normal adult zebrafish telencephalon, the proliferation of $\mathrm{PCNA}^{+} /$olig $2^{+}$RGs and OPCs was not observed in the ventricular zone or parenchymal area, respectively (Fig. $2 \mathrm{~B} \sim \mathrm{B}^{\prime}$ ). However, we observed an increased number of $\mathrm{PCNA}^{+} /$olig2 $2^{+} \mathrm{OPC}$ near the injury site in the telencephalic parenchyma, which suggests that OPCs in the lesioned hemisphere responded to injury and increased proliferation 4 dpl (Fig. 2C, C", E). Consistent with previous studies, which have shown that RGs in the LVZ increase proliferation to regenerate new neurons in the lesioned telencephalon [9], we also observed the increased proliferation of $\mathrm{S} 100 \mathrm{~b}^{+} \mathrm{RG}$ in the LVZ of the lesioned telencephalon $4 \mathrm{dpl}$ (Fig. 2B, C, yellow arrows). Interestingly, increased proliferation of olig $2^{+} \mathrm{RGs}$ was also detected in the MVZ of the lesioned adult zebrafish telencephalon $4 \mathrm{dpl}$ (Fig. 2C, C, F). Proliferation of OPCs and olig2 ${ }^{+}$RGs in the lesioned telencephalon decreased $21 \mathrm{dpl}$, indicating that the injury response had diminished at this stage (Fig. 2D F). Altogether, these data suggest that olig2 $2^{+}$RG in the MVZ and parenchymal 
A
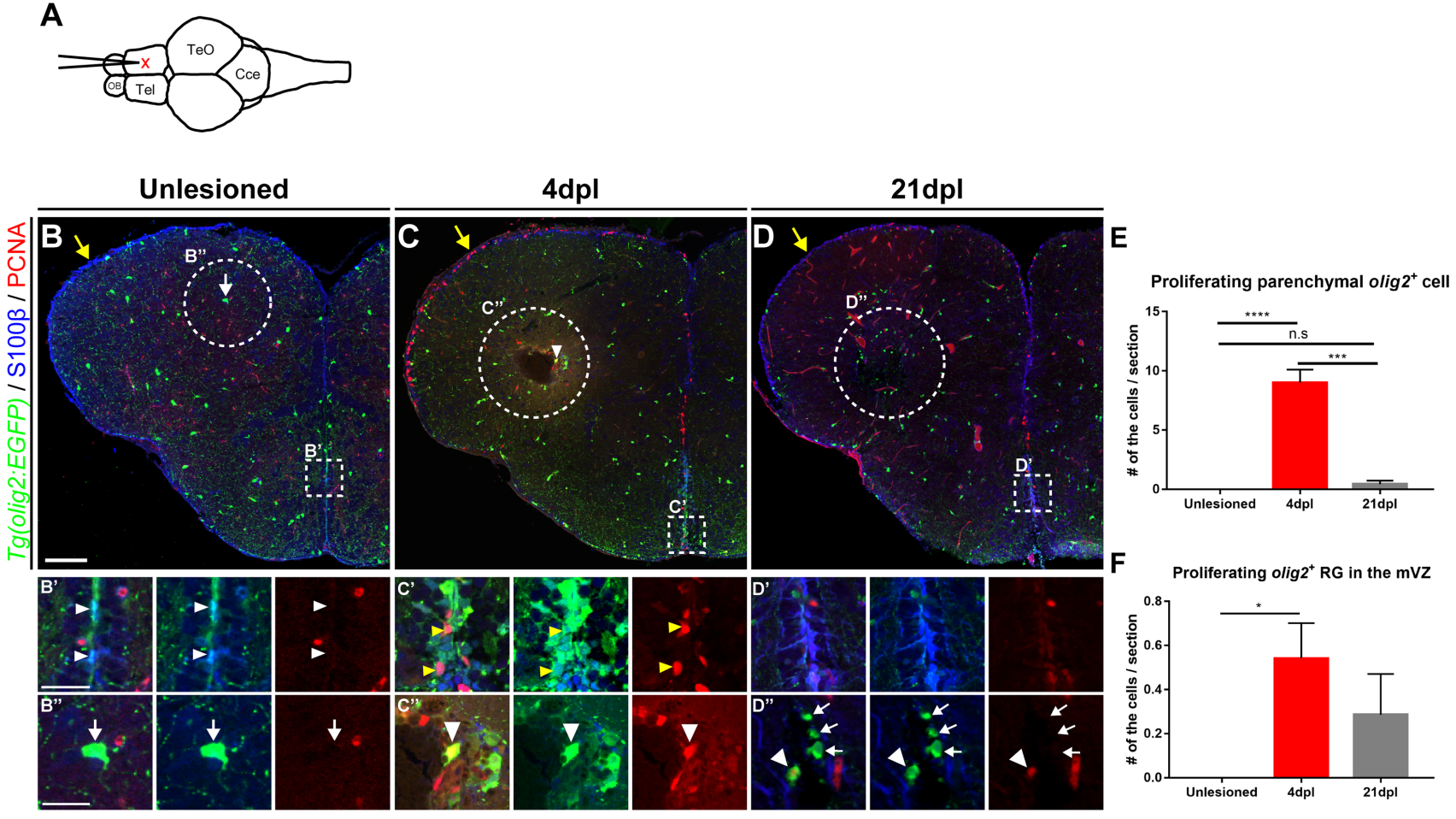

Proliferating olig2 ${ }^{+}$RG in the $\mathrm{mVZ}$

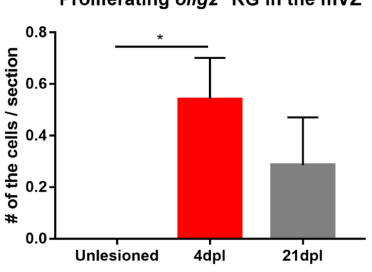

Fig. 2. Proliferation of Olig2 $2^{+}$G and parenchymal OPCs is increased in the injured hemisphere of the adult telencephalon. All panels show transverse sections of the telencephalon, with the dorsal side at the top. (A) Scheme depicting the stab wound paradigm in the adult zebrafish telencephalon. (B D) Labeling of adult $\operatorname{Tg}$ (olig2:EGFP) zebrafish with a proliferation marker (PCNA) and RG marker (S100 $\beta$ ) in the uninjured controls (B), 4 days postlesion $(\mathrm{dpl})(\mathrm{C})$, and $21 \mathrm{dpl}(\mathrm{D})$. Yellow arrows indicate the LVZ area of the injured hemisphere of the telencephalon. (B' D') The MVZ of the normal (B') and injured hemisphere (C, D') of the telencephalon. White arrowheads indicate olig2:EGFP ${ }^{+} / \mathrm{PCNA}^{-}$non-proliferating RG (B') and yellow arrowheads indicate olig2: $\mathrm{EGFP}^{+} / \mathrm{PCNA}^{+}$proliferating $\mathrm{RG}\left(\mathrm{C}^{\prime}\right)$. $\left(\mathrm{B}^{\prime} \sim \mathrm{C}^{\prime \prime}\right)$ The parenchymal area of the wildtype $\left(\mathrm{B}^{\prime \prime}\right)$ and injured hemispheres $\left(\mathrm{C}^{\prime \prime}, \mathrm{D}^{\prime \prime}\right)$ of the telencephalon. Arrows indicate non-proliferating OPCs (B", D") and arrowheads indicate proliferating OPCs (C", D") in the parenchyma. (E) Quantification of $\mathrm{S} 100 \beta^{-} / \mathrm{EGFP}^{+} / \mathrm{PCNA}^{+}$proliferating OPCs in the parenchymal area of the telencephalon $(\mathrm{n}=8$ sections from three zebrafish, Kruskal-Wallis test,

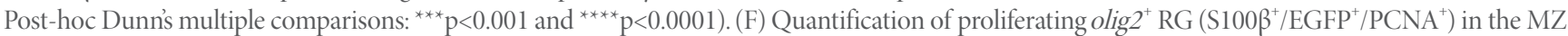
( $\mathrm{n}=8$ sections from three zebrafish, Kruskal-Wallis test, Post-hoc Dunn's multiple comparisons: $\left.{ }^{*} \mathrm{p}<0.05\right)$. Scale bars: B D, $100 \mu \mathrm{m} ; \mathrm{B} \sim \mathrm{D}$ ”, $25 \mu \mathrm{m}$. EGFP, enhanced green fluorescent protein; LVZ, lateral ventricular zone; MVZ, medial ventricular zone; OPCs, oligodendrocyte progenitor cells; PCNA, proliferating cell nuclear antigen; RG, radial glia.

OPCs respond to injury and temporarily increase proliferation.

To test the hypothesis that increased proliferation of OPCs and olig2 $2^{+} \mathrm{RG}$ cells results in the regeneration of new oligodendrocytes, which are marked by $m b p$ :EGFP fluorescence in $T g(m b p$ :EGFP) zebrafish, we performed a BrdU pulse-chase experiment with the $\operatorname{Tg}$ (olig2:Dsred2); $\operatorname{Tg}(\mathrm{mbp}$ :EGFP) line and traced the newborn progeny of $o l i g 2^{+}$proliferative cells in the lesioned telencephalon of adult zebrafish (Fig. 3). After the telencephalic injury, we incorporated BrdU for 4 days (Fig. 3A) and observed that, similar to PCNA-labeled cells, BrdU-labeled cells were also detected throughout the lesioned hemisphere including the LVZ, MVZ, and parenchyma $4 \mathrm{dpl}$ (Fig. 3B, C). However, $\mathrm{BrdU}^{+} / m b p$ : $\mathrm{EGFP}^{+}$new oligodendrocytes were not detected at this stage, indicating that there were no newly regenerated mature oligodendrocytes $4 \mathrm{dpl}$ (Fig. 3C, C, E). After the long-term BrdU pulse-chase experiments, a significant number of $\mathrm{BrdU}^{+} / m b p: \mathrm{EGFP}^{+} /$olig2:Dsred ${ }^{+}$regener- ated oligodendrocytes were observed in the parenchyma of the lesioned hemisphere $21 \mathrm{dpl}$ (Fig. 3D, D', E), indicating that newly generated oligodendrocytes differentiated into mature oligodendrocytes.

\section{Notch signaling is required for the proliferation of OPCs and olig2 ${ }^{+}$RGs in the lesioned telencephalon}

Previously, Notch signaling has been shown to play a crucial role in adult neurogenesis and injury-induced regeneration of neurons in the zebrafish telencephalon $[9,11]$. To study the role of Notch signaling in the injury-induced proliferation of olig2 ${ }^{+}$RGs and OPCs in adult zebrafish telencephalon, we inhibited Notch signaling in vivo through treatment with LY-411575, a pharmacological inhibitor of r-secretase that impairs the generation of the Notch intracellular domain, thus blocking Notch activation [22]. Inhibition of Notch signaling in normal adult zebrafish following 
A
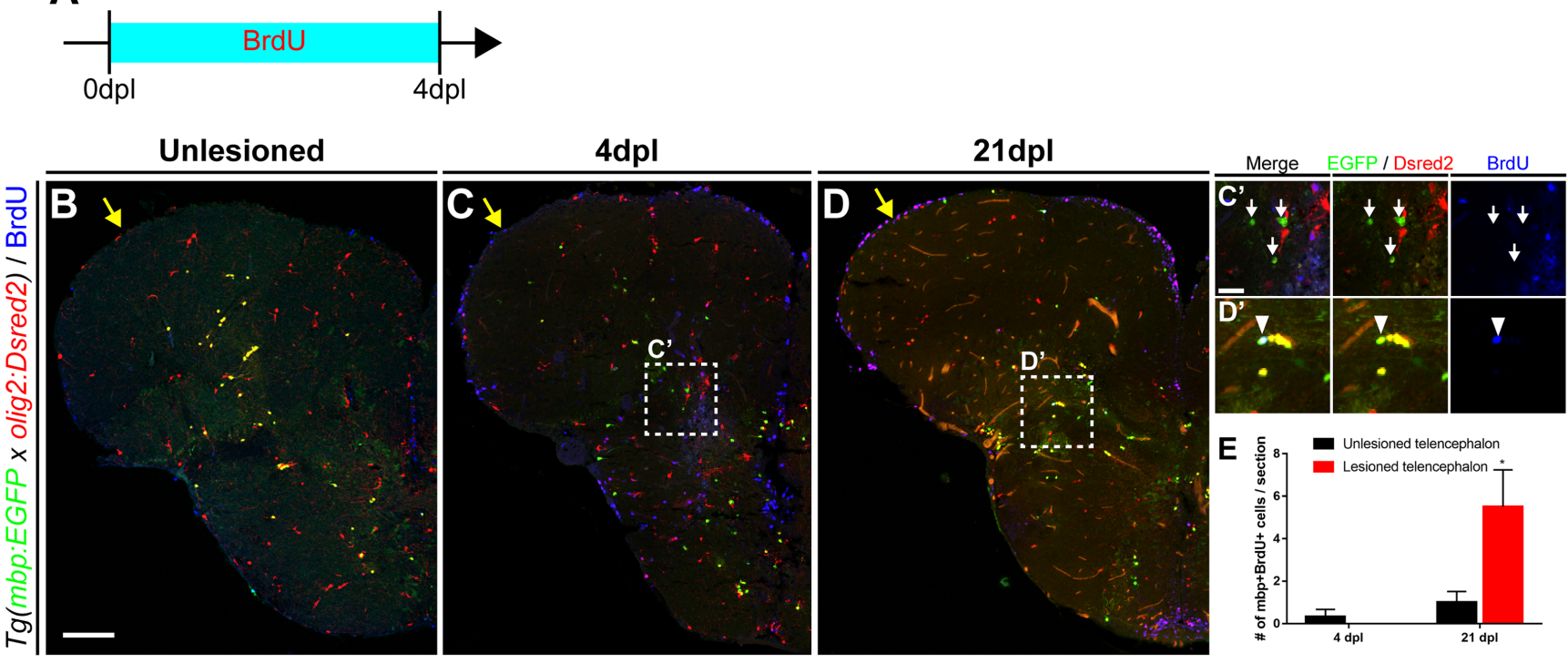

Fig. 3. Regeneration of mature oligodendrocytes in the injured telencephalon. All panels show transverse sections of the telencephalon of adult zebrafish, with the dorsal side at the top. (A) Scheme of BrdU treatment. (B D) Labeling of the telencephalon of $\operatorname{Tg}(\mathrm{mbp}$ :EGFP) adult zebrafish with antiBrdU antibody. Yellow arrows indicate the LVZ area of the control (B) and injured hemispheres of the telencephalon (C, D). (C', D') Boxed areas in C and $\mathrm{D}$ indicate the parenchymal area near the injury site. Arrows indicate $m b p$ : $\mathrm{EGFP}^{+} / \mathrm{BrdU}^{-}$pre-existing mature oligodendrocytes (C'), and arrowheads indicate $\mathrm{mbp}: \mathrm{EGFP}^{+} / \mathrm{BrdU}^{+}$newly generated mature oligodendrocytes (D'). (E) Quantification of newly generated $\mathrm{EGFP}^{+} / \mathrm{Dsred}^{+} / \mathrm{BrdU}^{+}$mature oligodendrocytes ( $\mathrm{n}=7$ sections from two zebrafish, Mann-Whitney $U$ test, ${ }^{\star} \mathrm{p}<0.05$ ). Scale bars: $\mathrm{B} \sim \mathrm{D}, 100 \mu \mathrm{m} ; \mathrm{C}^{\prime} \sim \mathrm{D}$; $25 \mu \mathrm{m}$. BrdU, bromodeoxyuridine; EGFP, enhanced green fluorescent protein; LVZ, lateral ventricular zone.

treatment with LY-411575 increased the number of $\mathrm{PCNA}^{+}$proliferating RG in the LZV and MZV of the telencephalon (Fig. 4A B", E). We also observed increased numbers of $\mathrm{PCNA}^{+} /$olig2 $2^{+} \mathrm{RG}$ in the MVZ of the LY-411575-treated zebrafish (Fig. 4A', B', G). These data indicate that Notch signaling inhibits proliferation of RG, including olig2 ${ }^{+} \mathrm{RG}$, in the normal telencephalon of adult zebrafish. Increased proliferation of RGs and olig2 ${ }^{+}$RGs was consistently observed in lesioned hemispheres of the adult telencephalon following treatment with LY-411575 (Fig. 4C D", E, G). Interestingly, proliferation of OPCs in normal and lesioned hemispheres of the adult telencephalon were also increased following treatment with LY-411575, suggesting that Notch signaling is also involved in the regulation of parenchymal OPC proliferation in the normal and injured telencephalon of adult zebrafish (Fig. 4A D, F). Taken together, these data suggest that Notch signaling is implicated in the regulation of the proliferation of RGs and OPCs in the normal and lesioned telencephalon.

In this study, we showed in vivo evidence for the first time that olig2 ${ }^{+}$RGs are responsible for the regeneration of oligodendrocytes in the injured telencephalon of adult zebrafish. Telencephalic injury induced the active proliferation of olig2 $2^{+}$RGs and parenchymal OPCs shortly after injury, that is, $4 \mathrm{dpl}$. Injury-induced proliferation of olig2 ${ }^{+}$RGs and OPCs was followed by the regen- eration of new oligodendrocytes $21 \mathrm{dpl}$, suggesting that olig2 ${ }^{+}$ RGs and pre-existing OPCs are responsible for the regeneration of oligodendrocytes in the injured telencephalon of adult zebrafish. A previous study has shown that neuronal regeneration was observed $4 \mathrm{dpl}$ [8], that is, before the timing of oligodendrocyte regeneration observed in our study. This indicates that neuronal regeneration is followed by oligodendrocyte regeneration after telencephalic injury. Together, our data indicate that olig2 $2^{+} \mathrm{RG}$ are neural stem cells and participate in the regeneration of oligodendrocytes along with parenchymal OPCs in the injured telencephalon of adult zebrafish.

In mammals, although genetic fate-mapping studies have demonstrated that parenchymal OPCs are the principal cell type responsible for oligodendrocyte regeneration in demyelinating diseases in the adult brain $[23,24]$, NSCs in the subventricular zone (SVZ) also have been shown to have the potential to produce oligodendrocytes after injury [25, 26]. The participation of SVZderived progenitors in the remyelination process has been demonstrated in several experimental mouse models of demyelination $[27,28]$. Taken together, these data suggest that, like zebrafish, olig2-expressing cells among the NSCs in the MVZ may be responsible for the regeneration of oligodendrocytes in mammals.

Moreover, we showed that blocking Notch signaling promotes 


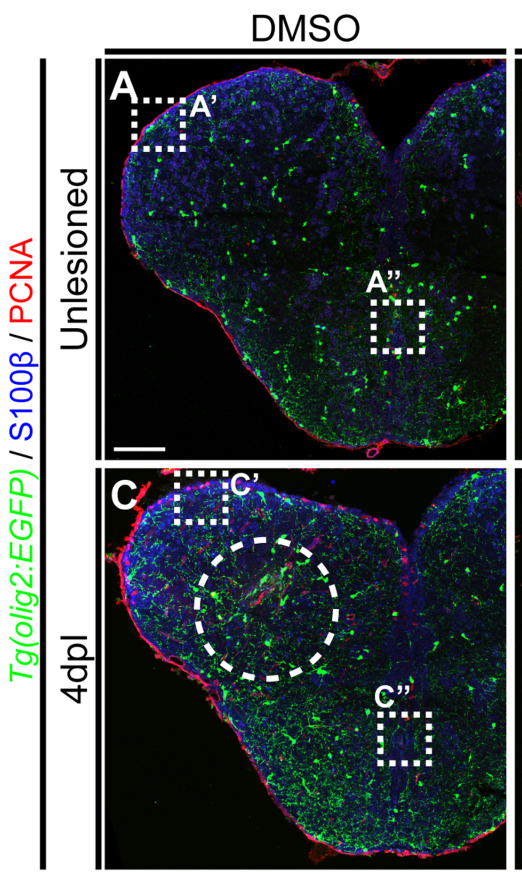

E Proliferating RG in the $\mathrm{mVZ}$

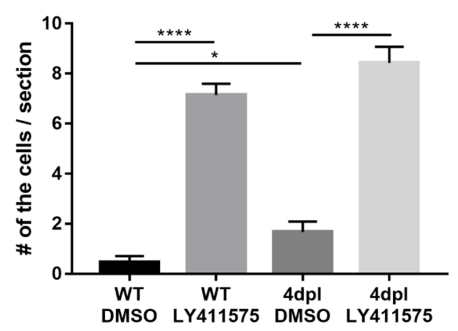

LY-411575
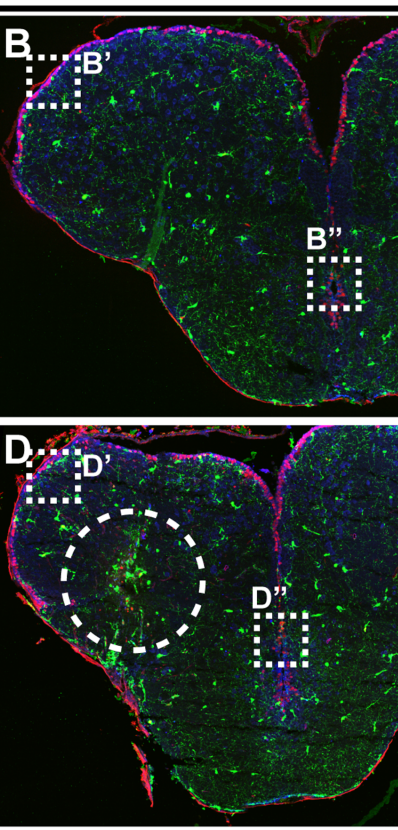

$\mathbf{F}$

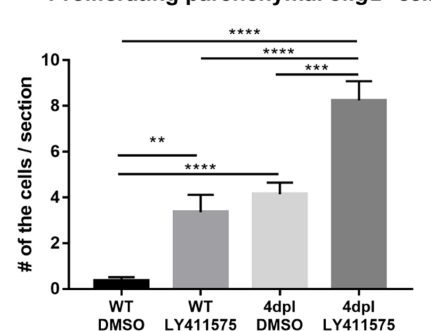

MVZ merge MVZ EGFP MVZ PCNA
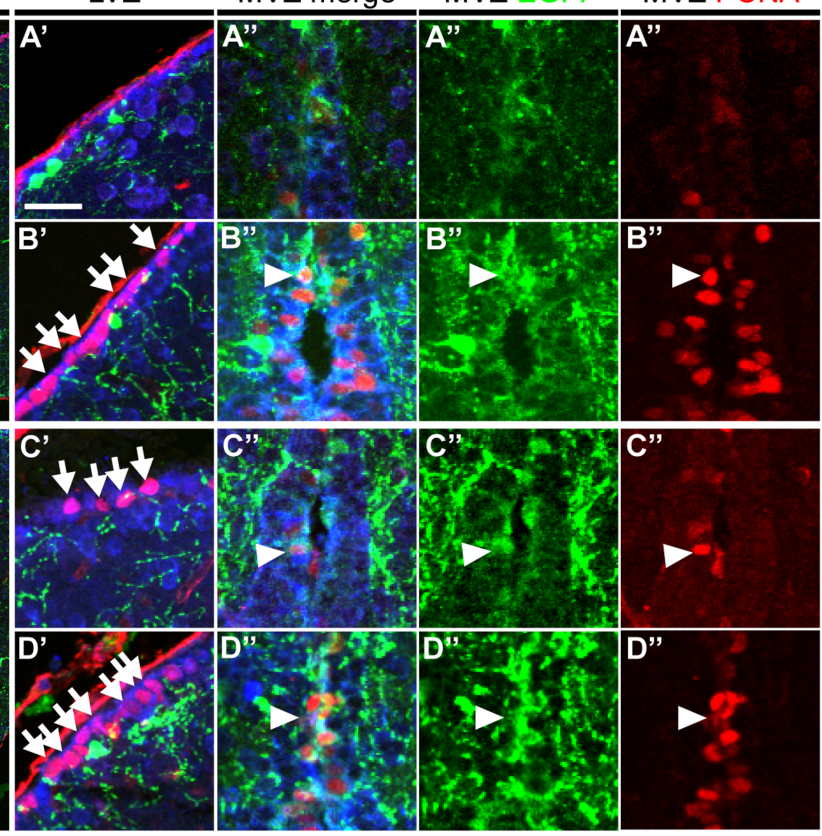

G

Proliferating olig2 ${ }^{+} \mathrm{RG}$ in the $\mathrm{mVZ}$

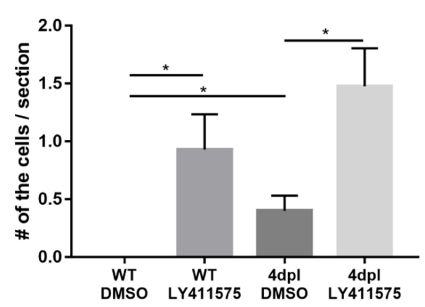

Fig. 4. Notch signaling is involved in the proliferation of olig2 $2^{+}$RG and parenchymal OPCs. All panels show transverse sections of the telencephalon, with the dorsal side at the top. (A D") Labeling of the telencephalon of $\operatorname{Tg}($ olig2:EGFP) adult zebrafish with anti-S100 $\beta$ and -PCNA antibodies. (A, B) Treatment of wildtypes with DMSO (A) and LY-411575 to inhibit Notch signaling (B). Boxed areas indicate the LVZ (A', B') and MVZ (A', B"). Arrows indicate $\mathrm{PCNA}^{+} / \mathrm{S} 100 \beta^{+}$proliferating RG (B'), and the arrowhead indicates $\mathrm{PCNA}^{+} / \mathrm{S} 100 \beta^{+} /$olig2:EGFP $\mathrm{EF}^{+}$proliferating RGs (B"). (C, D) Treatment of the injured telencephalon $4 \mathrm{dpl}$ with DMSO $(\mathrm{C})$ and LY-411575 to inhibit Notch signaling (D). Arrows indicate PCNA $/$ S100 $\beta^{+}$proliferating RGs $\left(\mathrm{C}^{\prime}, \mathrm{D}^{\prime}\right)$, and arrowheads indicate $\mathrm{PCNA}^{+} / \mathrm{S} 100 \beta^{+} /$olig2:EGFP ${ }^{+}$proliferating RGs $\left(\mathrm{C}^{\prime}, \mathrm{D}^{\prime \prime}\right)$. (E $\left.\sim \mathrm{G}\right)$ Quantification of proliferating $\mathrm{RGs}\left(\mathrm{S} 100 \beta^{+} / \mathrm{EGFP}^{+} / \mathrm{PCNA}{ }^{+}\right)$in the medial ventricular zone (E), proliferating parenchymal OPCs $\left(\mathrm{S} 100 \beta / \mathrm{EGFP}^{+} / \mathrm{PCNA}^{+}\right)(\mathrm{F})$, and proliferating olig2 $2^{+} \mathrm{RGs}\left(\mathrm{S} 100 \beta^{+} / \mathrm{EGFP}^{+} / \mathrm{PCNA}^{+}\right)$in the medial ventricular zone $(G)\left(n=9\right.$ sections from two zebrafish, Mann-Whitney $U$ test, ${ }^{*} p<0.05,{ }^{* *} p<0.01,{ }^{* * *} p<0.001$ and $\left.{ }^{* * * *} p<0.0001\right)$. Scale bars: A D, $100 \mu \mathrm{m} ; \mathrm{A}^{\prime} \sim \mathrm{D}^{\prime}, 25 \mu \mathrm{m}$. DMSO, dimethyl sulfoxide; EGFP, enhanced green fluorescent protein; LVZ, lateral ventricular zone; MVZ, medial ventricular zone; OPCs, oligodendrocyte progenitor cells; PCNA, proliferating cell nuclear antigen; RG, radial glia.

the proliferation of olig2 $2^{+}$RGs and OPCs in the normal and injured telencephalon of adult zebrafish, suggesting that Notch signaling is implicated in the regeneration of oligodendrocytes by regulating the proliferation of olig2 $2^{+}$RGs and pre-existing OPCs. Our data are also supported by a previous study which showed that NSCs transition between the quiescent and proliferating states according to the level of Notch activity and that Notch induction drives NSCs into a quiescence state; in contrast, blocking Notch induces the proliferation of quiescent NSCs and the subsequent generation of neurons in the normal telencephalon of adult zebrafish [29]. Taken together, Notch signaling is suggested to be involved in the regeneration of neurons and oligodendrocytes by regulating the quiescence status of NSCs in the telencephalon of adult zebrafish.

\section{ACKNOWLEDGEMENTS}

This research was supported by the National Research Foundation of Korea (NRF), funded by the Ministry of Science and ICT (NRF-2018R1C1B5086223, NRF-2019R1I1A1A01043985). 


\section{REFERENCES}

1. Lee Y, Morrison BM, Li Y, Lengacher S, Farah MH, Hoffman PN, Liu Y, Tsingalia A, Jin L, Zhang PW, Pellerin L, Magistretti PJ, Rothstein JD (2012) Oligodendroglia metabolically support axons and contribute to neurodegeneration. Nature 487:443-448.

2. Alizadeh A, Dyck SM, Karimi-Abdolrezaee S (2015) Myelin damage and repair in pathologic CNS: challenges and prospects. Front Mol Neurosci 8:35.

3. Adolf B, Chapouton P, Lam CS, Topp S, Tannhäuser B, Strähle U, Götz M, Bally-Cuif L (2006) Conserved and acquired features of adult neurogenesis in the zebrafish telencephalon. Dev Biol 295:278-293.

4. Grandel H, Kaslin J, Ganz J, Wenzel I, Brand M (2006) Neural stem cells and neurogenesis in the adult zebrafish brain: origin, proliferation dynamics, migration and cell fate. Dev Biol 295:263-277.

5. Pellegrini E, Mouriec K, Anglade I, Menuet A, Le Page Y, Gueguen MM, Marmignon MH, Brion F, Pakdel F, Kah O (2007) Identification of aromatase-positive radial glial cells as progenitor cells in the ventricular layer of the forebrain in zebrafish. J Comp Neurol 501:150-167.

6. Lam CS, März M, Strähle U (2009) gfap and nestin reporter lines reveal characteristics of neural progenitors in the adult zebrafish brain. Dev Dyn 238:475-486.

7. März M, Chapouton P, Diotel N, Vaillant C, Hesl B, Takamiya M, Lam CS, Kah O, Bally-Cuif L, Strähle U (2010) Heterogeneity in progenitor cell subtypes in the ventricular zone of the zebrafish adult telencephalon. Glia 58:870-888.

8. Kroehne V, Freudenreich D, Hans S, Kaslin J, Brand M (2011) Regeneration of the adult zebrafish brain from neurogenic radial glia-type progenitors. Development 138:4831-4841.

9. März M, Schmidt R, Rastegar S, Strähle U (2011) Regenerative response following stab injury in the adult zebrafish telencephalon. Dev Dyn 240:2221-2231.

10. Baumgart EV, Barbosa JS, Bally-Cuif L, Götz M, Ninkovic J (2012) Stab wound injury of the zebrafish telencephalon: a model for comparative analysis of reactive gliosis. Glia 60:343-357.

11. Kishimoto N, Shimizu K, Sawamoto K (2012) Neuronal regeneration in a zebrafish model of adult brain injury. Dis Model Mech 5:200-209.

12. Rothenaigner I, Krecsmarik M, Hayes JA, Bahn B, Lepier A, Fortin G, Götz M, Jagasia R, Bally-Cuif L (2011) Clonal analysis by distinct viral vectors identifies bona fide neural stem cells in the adult zebrafish telencephalon and characterizes their division properties and fate. Development 138:14591469 .

13. Shin J, Park HC, Topczewska JM, Mawdsley DJ, Appel B (2003) Neural cell fate analysis in zebrafish using olig2 BAC transgenics. Methods Cell Sci 25:7-14

14. Park HC, Shin J, Appel B (2004) Spatial and temporal regulation of ventral spinal cord precursor specification by Hedgehog signaling. Development 131:5959-5969.

15. Kim H, Shin J, Kim S, Poling J, Park HC, Appel B (2008) Notch-regulated oligodendrocyte specification from radial glia in the spinal cord of zebrafish embryos. Dev Dyn 237:2081-2089.

16. Park HC, Shin J, Roberts RK, Appel B (2007) An olig2 reporter gene marks oligodendrocyte precursors in the postembryonic spinal cord of zebrafish. Dev Dyn 236:3402-3407.

17. Jung SH, Kim S, Chung AY, Kim HT, So JH, Ryu J, Park HC, Kim CH (2010) Visualization of myelination in GFP-transgenic zebrafish. Dev Dyn 239:592-597.

18. Feng L, Hatten ME, Heintz N (1994) Brain lipid-binding protein (BLBP): a novel signaling system in the developing mammalian CNS. Neuron 12:895-908.

19. Chapouton P, Adolf B, Leucht C, Tannhäuser B, Ryu S, Driever W, Bally-Cuif L (2006) her5 expression reveals a pool of neural stem cells in the adult zebrafish midbrain. Development 133:4293-4303.

20. Ganz J, Kaslin J, Hochmann S, Freudenreich D, Brand M (2010) Heterogeneity and Fgf dependence of adult neural progenitors in the zebrafish telencephalon. Glia 58:13451363.

21. März M, Schmidt R, Rastegar S, Strähle U (2010) Expression of the transcription factor Olig2 in proliferating cells in the adult zebrafish telencephalon. Dev Dyn 239:3336-3349.

22. Zhang L, Song L, Terracina G, Liu Y, Pramanik B, Parker E (2001) Biochemical characterization of the gamma-secretase activity that produces beta-amyloid peptides. Biochemistry 40:5049-5055.

23. Tripathi RB, Rivers LE, Young KM, Jamen F, Richardson WD (2010) NG2 glia generate new oligodendrocytes but few astrocytes in a murine experimental autoimmune encephalomyelitis model of demyelinating disease. J Neurosci 30:16383-16390.

24. Zawadzka M, Rivers LE, Fancy SP, Zhao C, Tripathi R, Jamen F, Young K, Goncharevich A, Pohl H, Rizzi M, Rowitch DH, Kessaris N, Suter U, Richardson WD, Franklin RJ (2010) CNS-resident glial progenitor/stem cells produce Schwann cells as well as oligodendrocytes during repair of CNS demyelination. Cell Stem Cell 6:578-590. 
25. Ming GL, Song H (2011) Adult neurogenesis in the mammalian brain: significant answers and significant questions. Neuron 70:687-702.

26. Luo Y, Coskun V, Liang A, Yu J, Cheng L, Ge W, Shi Z, Zhang K, Li C, Cui Y, Lin H, Luo D, Wang J, Lin C, Dai Z, Zhu H, Zhang J, Liu J, Liu H, deVellis J, Horvath S, Sun YE, Li S (2015) Singlecell transcriptome analyses reveal signals to activate dormant neural stem cells. Cell 161:1175-1186.

27. Klingener M, Chavali M, Singh J, McMillan N, Coomes A, Dempsey PJ, Chen EI, Aguirre A (2014) N-cadherin promotes recruitment and migration of neural progenitor cells from the SVZ neural stem cell niche into demyelinated lesions. J Neurosci 34:9590-9606.

28. Samanta J, Grund EM, Silva HM, Lafaille JJ, Fishell G, Salzer JL (2015) Inhibition of Glil mobilizes endogenous neural stem cells for remyelination. Nature 526:448-452.

29. Chapouton P, Skupien P, Hesl B, Coolen M, Moore JC, Madelaine R, Kremmer E, Faus-Kessler T, Blader P, Lawson ND, Bally-Cuif L (2010) Notch activity levels control the balance between quiescence and recruitment of adult neural stem cells. J Neurosci 30:7961-7974. 